\title{
Guest editorial: Special issue on online decision making in multi-robot coordination
}

\author{
Jen Jen Chung ${ }^{1,2}$ (I) Jan Faigl ${ }^{3}\left(\mathbb{C} \cdot\right.$ Geoffrey A. Hollinger ${ }^{1}$
}

Published online: 26 February 2018

๑) Springer Science+Business Media, LLC, part of Springer Nature 2018

Online decision making is an important part of robotics problems in which mobile robots operate in unknown or partially known dynamic environments with the goal of acquiring information about some studied phenomena. This can be found in problems such as autonomous data collection, environmental monitoring, and robotic exploration missions that can be considered as variants of robotic information gathering. The key aspect of these problems is that the overall mission performance can only be evaluated after the mission is completed; however, the choice of which action to take at any time depends on local in-situ conditions that vary according to the information acquired during the mission.

This special issue aims at presenting the state-of-the-art in approaches to online decision making for coordinating a team of mobile robots to fulfill a global mission objective through the individual actions of each robot. The particular focus is on missions such as multi-robot exploration, persistent environmental monitoring, and adaptive information gathering. The fundamental challenge of these missions is that little or no information about the environment is known in advance. Therefore, one of the problems that must be addressed is how to trade-off exploration of the unknown parts of the environment to collect new information about the operational environment, and exploitation of the current knowledge acquired so far to improve the mission performance.

\section{Jen Jen Chung}

jenjen.chung@mavt.ethz.ch

Jan Faigl

faiglj@fel.cvut.cz

https://robotics.fel.cvut.cz/jf/

Geoffrey A. Hollinger

geoff.hollinger@oregonstate.edu

1 Oregon State University, 2000 SW Monroe Ave, Corvallis, OR 97330, USA

2 ETH Zurich, Leonhardstrasse 21, 8092 Zurich, Switzerland

3 Faculty of Electrical Engineering, Czech Technical University in Prague, Technická 2, 16627 Prague 6, Czech Republic
Following an open call for papers, the 12 selected articles (with a $50 \%$ acceptance rate out of 24 submissions) that comprise this special issue contain a common thread of distributed planning for multi-robot active perception, navigation, and manipulation. To solve these challenging problems, the authors leverage and build on techniques such as belief space planning, self-organizing maps, Gaussian Markov random fields, model-free control, homotopy constrained optimization, and team orienteering. Collectively these papers contribute a diverse set of tools for multi-robot planning and optimization in highly uncertain and dynamic environments.

A summary of each of the 12 articles is provided below.

Decentralized multi-robot belief space planning in unknown environments via identification and efficient re-evaluation of impacted paths (Regev and Indelman)

The first article provides a computationally efficient method for decentralized belief space planning with applications in multi-robot autonomous navigation in unknown environments. By identifying and only updating those paths that are impacted as a result of an update in the path of another robot, their method is able to achieve substantial planning time speed-ups compared to existing approaches.

Online planning for multi-robot active perception with self-organizing maps (Best, Faigl and Fitch)

This contribution presents a self-organizing map algorithm that jointly optimises the selection and sequence of viewpoint nodes to allocate to robots that are coordinating to perform active perception and data collection tasks. The authors show that their solution allows for efficient online replanning by adapting previous solutions as new information becomes available.

Searching and tracking people with cooperative mobile robots (Goldhoorn et al.)

This paper discusses a decentralized multi-robot approach to searching and tracking a human in a dynamic environment 
where individual robots share their observations and belief of the target location. The authors show that their approach is robust to communication drop-outs and noisy sensors that provide false detections.

Learning environmental fields with micro underwater vehicles: A path integral - Gaussian Markov random field approach (Kreuzer and Solowjow)

The authors provide an information theoretic framework that combines the path integral control with inference using Gaussian Markov Random Fields for navigating a micro AUV fleet to explore and monitor spatiotemporal flow fields. The proposed approach is designed to work well in communication-constrained environments such as coordinated underwater inspection.

Timed abstractions for distributed cooperative manipulation (Verginis and Dimarogonas)

The authors derive well-defined timed abstractions for the decentralized cooperative manipulation of a single object by multiple robots. They propose a distributed model-free control protocol for tracking the trajectory of the object and combine this with standard automata-based methods to allow for the development of complex shared manipulation tasks over time.

Reactive mission and motion planning with deadlock resolution avoiding dynamic obstacles (Alonso-Mora et al.)

This contribution designs a holistic synthesis approach to guarantee collision-free behaviors in dynamic environments with an arbitrary number of moving obstacles. The authors combine this with an automatic means for encoding tasks that resolve deadlock scenarios based on automaticallygenerated revisions to a specification. The problem addressed in this paper is similar to that of Gregoire, Čáp, and Frazzoli, which is also published in this issue.

Complete 3-D dynamic coverage in energy-constrained multi-UAV sensor networks (Bentz et al.)

The authors discuss an energy-aware deployment and guidance protocol for a multi-robot 3D dynamic coverage task. Their method redistributes robots based on their available energy and accounts for persistent battery replacements to ensure continuous coverage.

Reconfigurable multi-robot coordination with guaranteed convergence in obstacle cluttered environments under local communication (Vrohidis et al.)

This paper presents a distributed reconfiguration strategy for a networked team of robots navigating through cluttered environments. The proposed approach assures convergence to the desired goal location while obeying formation specifications and guaranteeing global connectivity across the team.

Strategies for coordinated multi-robot exploration with recurrent connectivity constraints (Banfi et al.)

The authors provide exploration strategies that allow robots to coordinate with teammates and form ad hoc networks that allow the relay of data back to a base station when making new observations. The main goal is to maintain situational awareness at the base station without sacrificing exploration efficiency of the distributed team.

Locally-optimal multi-robot navigation under delaying disturbances using homotopy constraints (Gregoire, Čáp and Frazzoli)

In this contribution, the authors design coordinated motion planning for multiple robots, specifically accounting for the possibility of disturbances that cause delays in the path execution of individual robots. The authors derive a maximal set of homotopic solutions in the coordination space and present a controller that guarantees the system remains within this set of solutions despite any delaying disturbance affecting the advancement of individual robots. The problem addressed in this paper is similar to that of Alonso-Mora et al., which is also published in this issue.

Multiple-place swarm foraging with dynamic depots (Lu, Hecker and Moses)

This paper presents a scalable, decentralized architecture for multi-robot foraging with multiple mobile depots where both the robots and depots move to optimize foraging rate. The spatially distributed design of the proposed algorithm reduces robot transport time as well as time spent applying collision avoidance maneuvers.

The Team Surviving Orienteers problem: routing teams of robots in uncertain environments with survival constraints (Jorgensen et al.)

The authors formalize the team surviving orienteers problem, an extension of the team orienteering problem where robots must traverse a graph with some risk of catastrophic failure along each edge. The goal of the team is to maximize the number of sites visited while maintaining the return probability of each robot above a specified survival threshold. The authors present an approximate greedy approach to this problem of survivability-aware multi-robot coordination.

This special issue grew from the series of workshops on "Online Decision Making in Multi-robot Coordination", organized by Jan Faigl, Geoffrey A. Hollinger, Jen Jen Chung, Olivier Simonin and François Charpillet, and held 
at the 2015 IEEE/RSJ International Conference on Intelligent Robots and Systems (October 2015 in Hamburg, Germany) and 2016 Robotics: Science and Systems Conference (June 2016 in Ann Arbor, Michigan). We would like to acknowledge the excellent work of the authors, reviewers and entire editorial team in helping us to curate this special issue. 